\title{
Inhalt $^{1}$
}

Vorwort

1. Einführung in die Thematik 9

$1.1 \quad$ Warum Frame-Semantik? 9

1.2 Ein Märchen - Semantik, wie sie am schönsten (und einfachsten) wär $\quad 12$

1.3 Zum Aufbau des vorliegenden Buches 19

2. Die Erfindung des Frame-Gedankens in der Linguistik -

Der Denkweg von Charles J. Fillmore 23

2.1 Was ist in einem Satz oder Text semantisch ,enthalten“? 26

2.2 Valenz-Modell und Kasus-Rahmen 34

Z.3 Zwischen Kasus-Grammatik und Lexikalischer Semantik:
Auf dem Weg zur Frame-Theorie

2.4 Die ,,scenes-and-frames “-Semantik 53

2.5 Die Aufgaben einer linguistischen Semantik 81

2.6 „Frame-Semantik“ und die Bedingungen des Verstehens:

2.7 Anwendungsbereiche und Leistungen des Frame-Modells 132

2.8 Ein „technisches“ Modell: Prädikative Frames (FrameNet) 140

2.9 Fillmores linguistische Frametheorie: Zusammenfassender Überblick 209

3. Die Begründung der Frame-Theorie in der Kognitionswissenschaft -

Die Frame-Idee bei Marvin Minsky 251

3.1 Kognitive Frames: Minskys Startschuss 252

3.2 Minskys Überlegungen zu Frames in Sprache und Textverstehen 264

3.3 Weitere Aspekte von Frames, Frame-Strukturen und -Aktivierung 273

3.4 Ein kognitives Modell des Denkens: Minskys „Society of mind“ 282

3.5 Zusammenfassung und Diskussion von Minskys Frame-Konzept 304

4. Frühe Anfänge und theoretische Fundierungen der Frame-Idee -

Die Schema- und Gedächtnistheorie von Frederic C. Bartlett 311

4.1 Psychologische Evidenzen für Frames: Bartletts „Remembering“ 311

4.2 Konsequenzen aus Bartletts Schema- und Gedächtnistheorie
für ein linguistisches Frame-Modell

5. Frame-theoretische Ausdifferenzierungen 336

5.1 Das Scripts-, Pläne-, Ziele-Modell von Schank \& Abelson 337

5.2 Systematisierungsversuche des Frame-Modells bei L. Barsalou 361

5.3 Diskussion der Modelle und Konsequenzen für die linguistische Semantik $\quad 414$

5.4 Zur weiteren Diskussion um Frames 420

1 Dieses Inhaltsverzeichnis führt nur die Hauptkapitel und Unterkapitel 1. Ordnung auf. Ein ausführliches Gesamt-Inhaltsverzeichnis mit sämtlichen Unterkapiteln 2. Ordnung findet sich am Ende des Bandes. 
6. Anwendungen und Weiterentwicklungen der Frame-Theorie in der linguistischen Semantik

6.1 Fillmores FrameNet 441

6.2 Lexikologisch-lexikographische Ansätze in Deutschland 451

6.3 Frame-Analysen in der Formalen Linguistik 495

6.4 Frame-Analyse als satz-, text- und kontext-semantische Methode 502

7. Frame-Semantik: Ein Arbeitsmodell 533

7.1 Sprachliche Frames oder kognitive Frames? Eine Entscheidung 533

Frames als Format der Organisation und Rekonstruktion
des verstehensrelevanten Wissens

7.3 Frame-Typen (I): Prädikative Frames vs. Konzept-Frames 550

7.4 Die innere Struktur von Frames 553

7.5 Merkmale von Frames:

Prototypikalität, Konventionalität, Default-Werte, Iterativität, Rekursivität, Vernetzbarkeit, Perspektivierung, Fokussierung und Frame-Dynamik 595

$\begin{array}{lll}7.6 & \text { Die Struktur von Frame-Systemen und -Netzen } & 627\end{array}$

7.7 Evokation oder Invokation von Frames? (Was gehört zu einem Frame?) 644

$\begin{array}{lll}7.8 & \text { Typen von Frames (II) und Frame-Aktivierung } & 670\end{array}$

7.9 Epistemische Prädikation als Grundstruktur von Frames

7.10 Ein Frame-gestütztes Modell des Sprachverstehens? $\quad 704$

$\begin{array}{lll}7.11 & \text { Frames praktisch: Modelle der Darstellung } & 705\end{array}$

7.12 Anwendungsmöglichkeiten der Frame-Analyse 742

8. Frame-Analyse und linguistische Epistemologie - Resümee und Ausblick 787

8.1 Kognitive Semantik: Ein Wort an die Gebildeten unter ihren Verächtern 788

8.2 Frame-Analyse als Teil einer linguistischen Epistemologie 805

8.3 Ausblick und Anschlussmöglichkeiten 813

8.4 Statt einer Zusammenfassung: 66 Thesen zu Frames und Frame-Semantik 818

$\begin{array}{lr}\text { Bibliographie } & 828\end{array}$

$\begin{array}{ll}\text { Anhang } & 844\end{array}$

$\begin{array}{ll}\text { Register } & 877\end{array}$

Detailliertes Gesamt-Inhaltsverzeichnis $\quad 884$ 